\title{
Connect medical education practices and research in Asia with the rest of world
}

Young-Mee Lee

Medical Education Unit, Department of Medical Humanities, Korea University College of Medicine, Seoul, Korea

Since its first issue in 1989, the Korean Journal of Medical Education (KJME) has rapidly grown in terms of both quantity and quality. It was indexed in MEDLINE in 2015 and in PubMed Central (PMC) in 2016. Since the March 2016 issue, KJME has published in English only, to expand its reach among international readers and to promote more active interactions with medical educators across the world. Now, KJME is waiting on the review results from the Scopus and Directory of Open Access Journals (DOAJ) databases. Such success, in a relatively short period, has been possible because of the medical educators and researchers who have cared deeply for KJME. However, as the new editor-in-chief of KJME, I would like to express my sincere respect and gratitude to my predecessor, Prof. Sun Kim, the former deputy editor Prof. Sun Hur, and the previous leadership of the Korean Society of Medical Educators. Without their leadership, passion, endless devotion, and contributions, KJME would not have reached its current position.

This March will mark the second year of KJME as an English-language publication. Starting the English edition was very challenging for both the authors and the KJME editorial board. However, the 2016 editorial board successfully brought out four issues. Our next goal is to be indexed in the Science Citation Index Expanded (SCIE). This is indeed one of the most challenging hurdles for the journal. To accomplish this ultimate goal, recently KJME have set a new editorial board member which consists of world renowned medical educators and young Korean medical education researchers. I would like to express my gratitude to new board members and those who have been retained for another year. I am especially grateful to Prof. Ronald Harden, a senior consultant.

Although KJME began its ambitious journey with a strong editorial board, the continued interest and contributions by our readers and authors have been of the utmost importance. As a professional medical education journal with a relatively long history in Asian countries, KJME needs support from readers not only in Korea but all over the world.

One of KJME's main objectives is to become a leading
Received: February 7, 2016 • Revised: February 17, 2016 • Accepted: February 17, 2016 Corresponding Author: Young-Mee Lee (http://orcid.org/0000-0002-4685-9465) Medical Education, Department of Medical Humanities, Korea University College of Medicine, 73 Inchon-ro, Seongbuk-gu, Seoul 02841, Korea

Tel: +82.2.2286.1098 Fax: +82.2.928.1647 email: ymleehj@korea.ac.kr
Korean J Med Educ 2017 Mar; 29(1): 3-5. https://doi.org/10.3946/kjme.2017.47 eISSN: 2005-7288

(C) The Korean Society of Medical Education. All rights reserved. This is an open-access article distributed under the terms of the Creative Commons Attribution Non-Commercial License (http:// creativecommons.org/licenses/by-nc/3.0/), which permits unrestricted non-commercial use, distribution, and reproduction in any medium, provided the original work is properly cited. 
Young-Mee Lee: Connect medical education practices and research in Asia with the rest of world

journal that can connect medical education practices and research in Asia with the rest of world. It is remarkable that despite the growing number of medical education papers, research, and reports from the Asian countries, we are still much far behind the West. This may be partly because Asian countries have taken to structured medical education research relatively recently. It is important to take into consideration the relatively limited educational resources - in terms of budgetary allocations, people, facilities, etc.-available in Asian medical schools, as compared to some advanced Western countries. Asian medical educators can devise a different approach to a difficult circumstance, to develop the best practices in medical education and research. The journal encourages papers that creatively interpret even wellknown facts from different perspectives in less resourceful settings. Medical education research conducted in Asian countries may be less rigorous than in countries with a long tradition in educational research and more resources. Even if a research or reported paper is less robust in study design or methods, but is novel and meaningful to international readers, KJME will be the best platform to connect authors to a worldwide audience. The journal looks forward to contributions from medical educators and researchers from all over the world.

In this issue, we selected four interesting original research papers on topics of growing interest-multiple mini-interviews, mentoring program evaluation tools, digital lectures, and students' patient-centeredness.

Kim et al. [1] examined the usability of multiple miniinterviews (MMI) in one Korean medical school. The MMI format emerged as a promising way to improve the student selection process even with limited educational resources [2,3]. Kim et al. reported their experience of MMI and its usefulness, including psychometric analysis. The strength of this study is the detailed description of how they constructed MMI stations in accordance with their institution's core values and educational goals. In addition, the authors reported an interesting finding: the participants' total MMI scores were not associated with their undergraduate grade point averages, nor their Korean medical school admission test (medical education eligibility test, MEET) scores. It indicates that the MMI test could measure a different domain of competence other than the cognitive, and it can be valued as an assessment tool for evaluating different aspects.

Ho et al. [4] introduced an evaluation tool for mentoring programs, called the Korean version of Noe's evaluation. As the authors mention in their paper, mentoring programs have been increasingly introduced in Korean medical colleges in recent years. This phenomenon is partly thanks to the accreditation system for continuous quality improvement in Korean medical schools. More importantly, the awareness in medical colleges and among medical educators about psychosocial support, academic advice, and career development counseling has increased, and most medical colleges now regard such student services as the university's responsibility. The authors' Korean version of Noe's evaluation is a timely article, published in KJME to meet the needs of those who want to evaluate the quality of mentoring programs. Compared to the original version of Noe's evaluation, an additional factor-"friendship"was explored by the authors. This may reflect a cultural difference or might be confined to this particular study. The methods of this study can provide useful information to researchers abroad trying to develop similar tools with their own vocabulary, or for a comparative study in different educational settings and cultures.

The authors from Mahsa University, Malaysia [5] developed a digital lecture on anatomy and examined the level of students' learning and attitudes towards the teaching and learning process when using digital lectures 
to teach gross anatomy to year 1 medical students. The authors compared the end-of-module summative test score between two different year cohorts, which were a didactic versus a digital lecture group. The authors reported a significant difference in test scores between the two cohorts. However, it is difficult to reach precise conclusions about the greater effectivity of the digital lecture on students' learning the same content because the study did not consider other possible contributing factors that might have affected the test results. In considering the paradigm shift in education towards digital learning, such as "flipped learning," diverse trials in new instructional methods should be carried out in every field of medical education.

Hur et al. [6] provided findings in agreement with the well-known difference in perspectives between patients and care providers. Medical educators should share experience and strategies which can narrow the difference in perspectives and needs between patients and doctors including medical students, who will be future doctors.

ORCID: Young-Mee Lee:

http://orcid.org/0000-0002-4685-9465

Acknowledgements: None.
Funding: None.

Conflicts of interest: None.

\section{References}

1. Kim KJ, Nam KS, Kwon BS. The utility of multiple mini-interviews: experience of a medical school. Korean J Med Educ 2017; 29: 7-14.

2. Lee HJ, Park SB, Park SC, Park WS, Ryu SW, Yang JH, Na S, Won JY, Chae GB. Multiple mini-interviews as a predictor of academic achievements during the first 2 years of medical school. BMC Res Notes 2016; 9: 93.

3. Kim DH, Hwang J, Kim EJ, Yoon HB, Shin JS, Lee S. How different are premedical freshmen who enter after introducing a multiple mini-interview in a medical school? Korean J Med Educ 2014; 26: 87-98.

4. Ho Y, Kwon OY, Park SY, Yoon TY, Kim YE. Reliability and validity test of the Korean version of Noe's evaluation. Korean J Med Educ 2017; 29: 15-26.

5. Singh A, Min AK. Digital lectures for learning gross anatomy: a study of their efficacy. Korean J Med Educ 2017; 29: 27-32.

6. Hur Y, Cho AR, Choi CJ. Medical students' and patients' perceptions of patient-centred attitude. Korean J Med Educ 2017; 29: 33-39. 